\title{
RADIALLY SYMMETRIC DISTRIBUTION OF MATTER
}

\author{
A. L. MEHRA
}

(Received 24 June 1965)

\section{Summary}

In this paper a solution of the Einstein field equations for a spherically symmetric distribution of a perfect fluid of variable density has been obtained.

\section{Introduction}

For the line element given by

$$
d s^{2}=-e^{\lambda} d r^{2}-r^{2} d \theta^{2}-r^{2} \sin ^{2} \theta d \phi^{2}+e^{\nu} d t^{2},
$$

Schwarzschild [1] has obtained the exterior solution

$$
e^{v}=1-2 m / r, \quad e^{\lambda}=(1-2 m / r)^{-1},
$$

and interior solution

$$
e^{\nu}=\frac{1}{4}\left[3\left(1-\frac{8 \pi \rho a^{2}}{3}\right)^{\frac{1}{2}}-\left(1-\frac{8 \pi \rho r^{2}}{3}\right)^{\frac{1}{2}}\right]^{2}, \quad e^{\lambda}=\left(1-\frac{8 \pi \rho r^{2}}{3}\right)^{-1},
$$

in which $m$ and $\rho$ respectively denote the mass and density inside a fluid sphere The solutions (1.2) and (1.3) obtained were based on the following assumptions:

(A) The density of fluid inside the sphere is constant and is zero outside the sphere.

(B) The matter comprises of a perfect fluid at rest inside the sphere and there is no matter out side the sphere.

(C) The pressure in zero at the surface of the sphere and is finite and positive inside the sphere.

(D) The graviational potentials of the exterior and interior solutions are continuous at the boundary of the sphere.

In the present paper the same problem of a spherical mass of a perfect fluid, with the density variable distribution inside the sphere has been studied. The variation of density has been arbitrarily taken as

$$
\rho=\rho_{C}\left(1-\frac{r^{2}}{a^{2}}\right)
$$


where $\rho_{C}$ is the density at the centre of the sphere. The law of density given by (1.4) has been taken to ensure that the density vanishes at the surface. The study with the variable density has been carried out earlier by Max Wyman [2]. He assumed the law of density to be $\rho=a r^{N-2}$, where $a$ and $N$ are constants. The present study differs from his investigation in that the density is zero at the surface in the present case.

\section{Solution of the field equations}

Since the matter comprises of a perfect fluid at rest, the components of energy momentum tensor $T^{\mu \nu}$ satisfy the following equations:

$$
T_{1}^{1}=T_{2}^{2}=T_{3}^{3}=-p, \quad T_{4}^{4}=\rho, \quad T_{j}^{i}=0, \quad i \neq j,
$$

where $p$ and $\rho$ are proper pressure and density of the fluid respectively.

The field equations for the line-element (1.1) are

$$
\begin{aligned}
& 8 \pi p=e^{-\lambda}\left(\frac{\nu^{\prime}}{r}+\frac{1}{r^{2}}\right)-\frac{1}{r^{2}}, \\
& 8 \pi p=e^{-\lambda}\left(\frac{\nu^{\prime \prime}}{2}-\frac{\nu^{\prime} \lambda^{\prime}}{4}+\frac{\nu^{\prime 2}}{4}+\frac{\nu^{\prime}-\lambda^{\prime}}{2 r}\right), \\
& 8 \pi \rho=e^{-\lambda}\left(\frac{\lambda^{\prime}}{r}-\frac{1}{r^{2}}\right)+\frac{1}{r^{2}} .
\end{aligned}
$$

In the above equations prime notation denotes differentiation with respect to $r$.

Equation (2.4) with equation (1.4), can be integrated to give

$$
e^{-\lambda}=1-\frac{8 \pi \rho_{C}}{15}\left(5 r^{2}-\frac{3 r^{4}}{a^{2}}\right)+\frac{C}{r},
$$

where $C$ is a constant of integration. In order to avoid a singularity at the origin $C$ should be zero. Therefore, taking $C=0$, equation (2.5) reduces to

$$
e^{-\lambda}=1-\frac{8 \pi \rho_{C}}{15}\left(5 r^{2}-\frac{3 r^{4}}{a^{2}}\right) \text {. }
$$

From equations (2.3) and (2.2), we find that the equation to determine $v$ is

$$
\begin{gathered}
{\left[1-\frac{8 \pi a^{2} \rho_{C}}{15}\left(5 x-3 x^{2}\right)\right]\left(2 r^{2} v^{\prime \prime}+v^{\prime 2} r^{2}\right)-r v^{\prime}\left(2-\frac{16 \pi a^{2} \rho_{C}}{5} x^{2}\right)} \\
+\frac{32 \pi a^{2} \rho_{C}}{5} x^{2}=0
\end{gathered}
$$


where $x=r^{2} / a^{2}$. The substitution

$$
v=2 \log y
$$

reduces equation $(2.7)$ to

(2.9) $4\left[1-\frac{8 \pi a^{2} \rho_{C}}{15}\left(5 x-3 x^{2}\right)\right] \frac{d^{2} y}{d x^{2}}+\frac{16 \pi a^{2} \rho_{C}}{15}(6 x-5) \frac{d y}{d x}+\frac{8 \pi a^{2} \rho_{C}}{5} y=0$.

Again by substituting

$$
z=\log \left[x-\frac{5}{6}+\left(x^{2}-\frac{5}{3} x+\frac{5}{8 \pi a^{2} \rho_{C}}\right)^{\frac{1}{2}}\right]
$$

in equation (2.9), we find that equation (2.9) reduces to

$$
\frac{d^{2} y}{d z^{2}}+\frac{y}{4}=0 \text {. }
$$

The solution of equation (2.11) is

$$
y=C_{1} \cos \frac{1}{2} z+C_{2} \sin \frac{1}{2} z,
$$

where $C_{1}$ and $C_{2}$ are the constants of integration; therefore from (2.8) we have

$$
e^{v}=\left[C_{1} \cos \frac{1}{2} z+C_{2} \sin \frac{1}{2} z\right]^{2} .
$$

From equations $(2.2),(2.6)$ and $(2.13)$, we find that the pressure at any point is given by

$$
\begin{aligned}
8 \pi p= & \frac{4}{a}\left(\frac{2 \pi \rho_{C}}{5}\right)^{\frac{1}{2}}\left[1-\frac{8 \pi a^{2} \rho_{C}}{15}\left(5 x-3 x^{2}\right)\right]^{\frac{1}{2}}\left[\frac{C_{2}-C_{1} \tan z / 2}{C_{1}+C_{2} \tan z / 2}\right] \\
& -\frac{8 \pi \rho_{C}}{15}(5-3 x) .
\end{aligned}
$$

By means of equations (2.6), (2.13), (2.14), (1.2) and boundary conditions (C) and (D), we get

$$
\begin{aligned}
m & =\frac{8 \pi a^{3} \rho_{C}}{15}, \\
C_{1} & =\left(1-\frac{16 \pi a^{2} \rho_{C}}{15}\right)^{\frac{1}{2}} \cos \frac{1}{2} z_{1}-\frac{2 a}{3}\left(\frac{2 \pi \rho_{C}}{5}\right)^{\frac{1}{2}} \sin \frac{1}{2} z_{1}, \\
C_{2} & =\left(1-\frac{16 \pi a^{2} \rho_{C}}{15}\right)^{\frac{1}{2}} \sin \frac{1}{2} z_{1}+\frac{2 a}{3}\left(\frac{2 \pi \rho_{C}}{5}\right)^{\frac{1}{2}} \cos \frac{1}{2} z_{1},
\end{aligned}
$$

where

$$
z_{1}=\log \left[\frac{1}{6}+\left(\frac{5}{8 \pi a^{2} \rho_{C}}-\frac{2}{3}\right)^{\frac{1}{2}}\right]
$$


The complete interior solution of line element (1.1) is, therefore,

(2.17a) $e^{\lambda}=\left[1-\frac{8 \pi \rho_{C}}{15}\left(5 r^{2}-\frac{3 r^{4}}{a^{2}}\right)\right]^{-1}$,

(2.17b) $e^{y}=\left[\left(1-\frac{16 \pi a^{2} \rho_{C}}{15}\right)^{\frac{1}{2}} \cos \left(\frac{z_{1}-z}{2}\right)-\frac{2 a}{3}\left(\frac{2 \pi \rho_{C}}{5}\right)^{\frac{1}{2}} \sin \left(\frac{z_{1}-z}{2}\right)\right]^{2}$,

where $z_{1}$ and $z$ are given by $(2.16)$ and (2.10) respectively.

To get a real solution from equations $(2.16)$ and $(2.15 \mathrm{a})$, we must hav

$$
a^{2}<\frac{9}{10 \pi \rho_{C}}, \quad m<\frac{12 a}{25},
$$

which give an upper limit of the possible size for given density and o the mass for given radius.

My thanks are due to Professor R. S. Kushwaha and Dr P. K. Bhati; for their kind interest in this paper.

\section{References}

[1] Tolman, R. C., Relativity thermodynamics and cosmology, Oxford Univ. Press 1962 p. 245.

[2] Max Wyman, Phys. Rev. 75 (1949), 1930.

Department of Mathematics

University of Jodhpur

Jodhpur 